\title{
The effect of strut thickness on shear stress distribution in a preclinical model
}

\author{
Erhan Tenekecioglu $^{1} \cdot$ Ryo Torii $^{2} \cdot$ Christos Bourantas $^{3,4} \cdot$ Yosuke Miyazaki $^{1}$. \\ Carlos Collet $^{5} \cdot$ Rasha Al-Lameé $^{6} \cdot$ Kadem Al-Lameé $^{7}$ - Yoshinobu Onuma ${ }^{1}$. \\ Patrick W. Serruys ${ }^{1,6,8}$
}

Received: 14 May 2017 / Accepted: 17 May 2017 / Published online: 31 May 2017

(c) The Author(s) 2017. This article is an open access publication

Keywords Bioresorbable scaffold $\cdot$ Local hemodynamics $\cdot$ Endothelial shear stress $\cdot$ Strut design

\section{Case report}

In-vitro and in-silico studies have shown that stent design and struts thickness introduce significant changes in local hemodynamics. The protruding struts disrupt the laminar flow with the impacts of strut thickness and strut shape. Angiographic and optical coherence tomography (OCT) data were implemented to reconstruct three-dimensional (3D) geometry of the right coronary artery (RCA) of a healthy mini-swine implanted with $3.0 \times 14 \mathrm{~mm}$ ArterioSorb (Arterius, UK) with 95 micron $(\mu \mathrm{m})$ strut thickness in proximal segment (final post-dilatation mean lumen

Erhan Tenekecioglu and Ryo Torii have contributed equally to this work.

Patrick W. Serruys

patrick.w.j.c.serruys@gmail.com

1 Department of Cardiology, Erasmus University Medical Centre, Thoraxcenter, Rotterdam, The Netherlands

2 Department of Mechanical Engineering, University College London, London, UK

3 Department of Cardiology, University College of London Hospitals, London, UK

4 Barts Heart Centre, Barts Health NHS Trust, London, UK

5 Department of Cardiology, Academic Medical Center, University of Amsterdam, Amsterdam, The Netherlands

6 Imperial College London, London, UK

7 Arterius, Leeds, UK

8 University of Melbourne, Melbourne, Australia diameter: $2.78 \mathrm{~mm}$ ) and $3.0 \times 14 \mathrm{~mm}$ ArterioSorb with $120 \mu \mathrm{m}$ strut thickness in mid-segment (final post-dilatation mean lumen diameter: $2.80 \mathrm{~mm}$ ) of the vessel.

During computational fluid dynamic (CFD) study, endothelial shear stress (ESS) was quantified in the scaffolded segment around the circumference of the lumen per $5^{\circ}$-subunit and along the axial direction per $200 \mu \mathrm{m}$ interval [1]. Median ESS was lower in the distal scaffold (ArterioSorb-120 $\mu \mathrm{m})[1.17(0.78-1.55) \mathrm{Pa}]$ than in the proximal scaffold (ArterioSorb-95 $\mu \mathrm{m})$ [1.25 (0.92-1.88) Pa] in Newtonian steady flow simulation ( $p<0.0001) .37 .4 \%$ of the scaffolded surface in ArterioSorb-120 $\mu \mathrm{m}$ and $32.6 \%$ in ArterioSorb-95 $\mu \mathrm{m}$ was exposed to a low $(<1 \mathrm{~Pa})$ atheropromoting ESS (Fig. 1). The p-value is based on $5^{\circ}$-subunit $(n=15,984)$ analysis using mixed effects regression model.

The difference in ESS may stem from strut thickness, luminal diameter, vessel curvature and flow boundary conditions. In the present case, after excluding other factors, lower ESS in ArterioSorb-120 $\mu \mathrm{m}$ is presumably ascribed to the thicker struts (Fig. 1). However, even in ArterioSorb-120 $\mu \mathrm{m}$, the shear stress was not as low as reported in Absorb BVS (Median ESS:0.57 Pa) [2] which should be attributed to the thinner struts of ArterioSorb. 


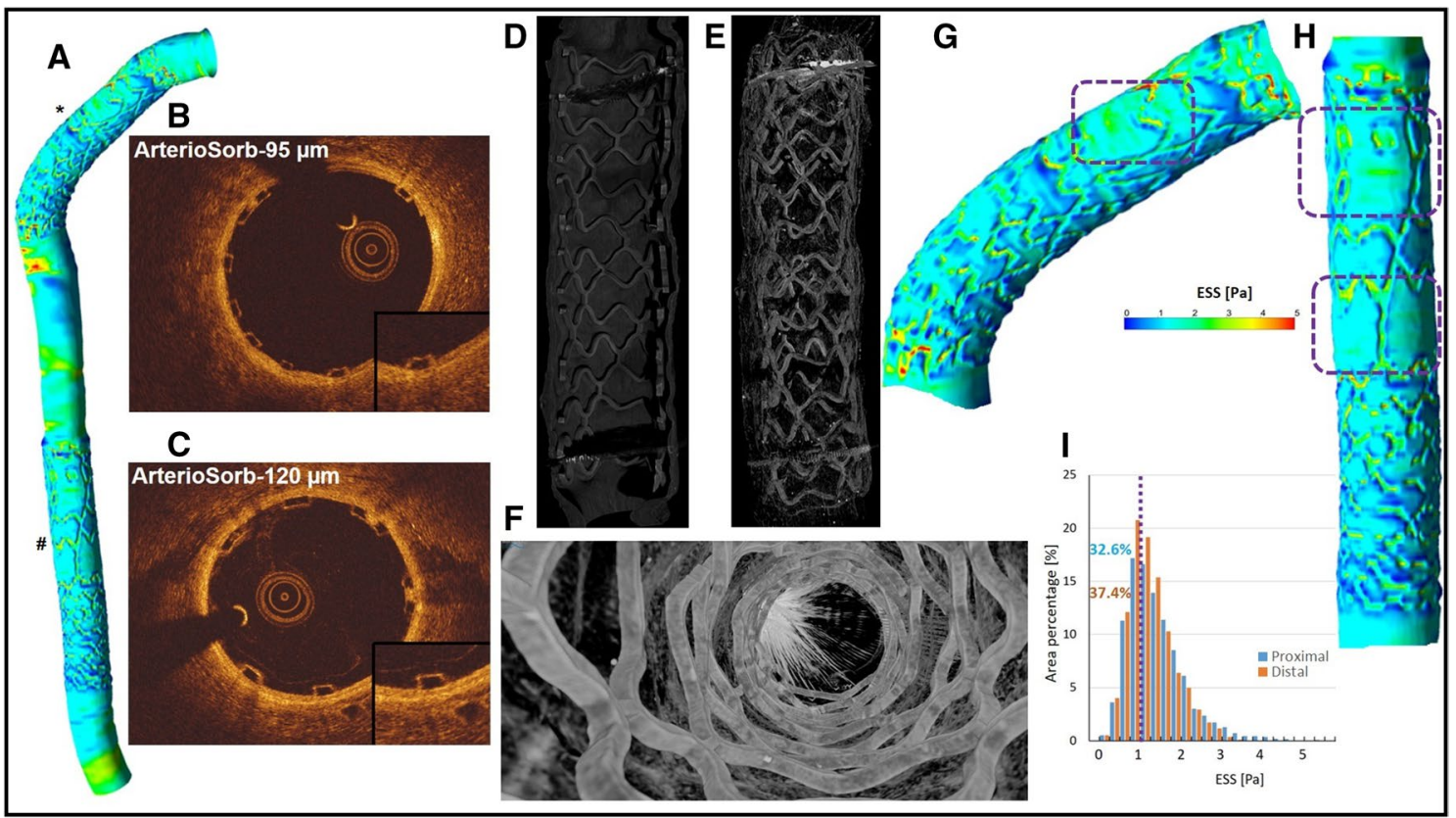

Fig. 1 The CFD model of the two ArterioSorb scaffolds (*ArterioSorb-95 $\mu \mathrm{m}$, \#ArterioSorb-120 $\mu \mathrm{m}$ ) with different strut thicknesses in RCA (a). OCT demonstrates well apposed struts in ArterioSorb-95 $\mu \mathrm{m}$ (b) and in ArterioSorb-120 $\mu \mathrm{m}$ (c). Micro-computed tomography shows the scaffold architectures without any discontinuity in ArterioSorb-95 $\mu \mathrm{m}(\mathbf{d})$ and in ArterioSorb-120 $\mu \mathrm{m}(\mathbf{e}, \mathbf{f})$. In

\section{Compliance with ethical standards}

Disclosures E. Tenekecioglu has a research grant from TUBITAK (The Scientific Council of Turkey). P.W. Serruys is a member of the International Advisory Board of Abbott Vascular. Y. Onuma is a member of the International Advisory Board of Abbott Vascular.

Research involving human and animal participants All applicable international and institutional guidelines for the care and use of animals were followed.

Open Access This article is distributed under the terms of the Creative Commons Attribution 4.0 International License (http:// creativecommons.org/licenses/by/4.0/), which permits unrestricted use, distribution, and reproduction in any medium, provided you give proximal (g) and the distal scaffolds (h), there are wide zones with smooth surfaces (dotted zones) which are considered to be the result of cardiac motion during the OCT pullback. The histograms (i) demonstrate the percentages of the vessel surface exposed to low-ESS $(<1 \mathrm{~Pa})$ in scaffolded vessel segments

appropriate credit to the original author(s) and the source, provide a link to the Creative Commons license, and indicate if changes were made.

\section{References}

1. Tenekecioglu E, Torii R, Bourantas C et al (2016) Preclinical assessment of the endothelial shear stress in porcine-based models following implantation of two different bioresorbable scaffolds: effect of scaffold design on the local haemodynamic micro-environment. EuroIntervention 12(10):1296

2. Tenekecioglu E, Torii R, Bourantas C et al (2017) Assessment of the hemodynamic characteristics of Absorb BVS in a porcine coronary artery model. Int J Cardiol 227:467-473 